ISBN 978-981-14-1684-2

Proceedings of 2019 the 9th International Workshop on Computer Science and Engineering

(WCSE 2019 SUMMER)

Hong Kong, 15-17 June, 2019, pp. 329-336

doi: $10.18178 /$ wcse. 2019.06 .050

\title{
Aviation Surveillance Information Fusion Technology Based on Recurrent Neural Network
}

\author{
Zhanchun $\mathrm{Gao}^{1}$, Anyu Song ${ }^{1}$ \\ ${ }^{1}$ School of Computer Science \\ Beijing University of Posts and Telecommunications, China
}

\begin{abstract}
Aviation surveillance information fusion is aimed at merging the detection data from multiple sources of the same target aircraft to obtain more accurate monitoring information, including aircraft position, heading, acceleration and other information. The traditional Kalman filter-based fusion technology has shortcomings, such as poor integration in the maneuvering state, and it takes a lot of manpower and material resources to repeat the adjustment. Therefore, this paper uses the recurrent neural network to conduct the experiment of aviation surveillance information fusion. Firstly, the recurrent neural network is used to identify the maneuver state of the aircraft, and the weighted least squares method is used to predict the position of the aircraft according to the maneuvering state, so as to obtain the monitoring information of each radar at the same time. After that, the recurrent neural network model is used to fuse the monitoring information of multiple radars. The experimental results show that the maneuvering state discriminant model based on recurrent neural network can effectively identify the maneuvering state. The least square method based on maneuvering state can accurately predict the position of the aircraft. The aeronautical surveillance information fusion model based on recurrent neural network can also obtain more accurate fusion results. The whole process includes four parts: preprocessing, maneuver status discrimination, position prediction and information fusion. The total time is about $500 \mathrm{~ms}$.
\end{abstract}

Keywords: Maneuvering state, Position prediction, Aviation surveillance information fusion, RNN.

\section{Introduction}

The role of the aviation surveillance system is to monitor and direct air traffic. The aviation surveillance system will display the aircraft's location, flight number, planned route, etc. to the controller. The controller will control the flight activity based on this information to ensure the flight safety of the aircraft [1]. The aeronautical surveillance information fusion technology studied in this paper is an important part of the aviation surveillance system. It is responsible for obtaining more accurate aircraft position information by processing the original monitoring information [2]. Since the original monitoring information is affected by various factors and the aircraft position information is not accurate, the result obtained by fusing the multisource information can be more advantageous than from a single information source [3]. In the field of aviation surveillance, the FAA's future aviation surveillance systems are divided into four geographic categories: ocean, domestic routes, terminal areas and ground. The domestic routes and terminal areas covered in this paper use ADS-B broadcast and secondary radar backup as the monitoring information source [4]. The secondary radar measurement error is mainly divided into the angular accuracy error and the ranging accuracy error [5]. Compared with the secondary radar signal, there is no measurement error in the ADS-B signal. The working principle of the ADS-B is that the airborne equipment receives the GPS signal for realtime positioning, and broadcasts the information such as the aircraft position at a time interval of one second. The surrounding aircraft and ground base stations will receive such data [6]. Although the ADS-B signal has

\footnotetext{
${ }^{1}$ Corresponding author:(Zhanchun Gao); Tel.: (+86-13701053226);
}

E-mail address: (gaozc@bupt.edu.cn). 
higher accuracy, the current domestic ADS-B ground station has not been fully covered, so it will still use two kinds of monitoring information to conduct air traffic.

At present, the core algorithm of the monitoring information fusion subsystem in the aviation monitoring system is the Kalman filtering algorithm [7]. Since its introduction in the 1960s, the Kalman filter has been widely used as a new filtering method in the fields of control, tracking and measurement. Due to the unbiased optimal estimation of the Kalman filter and the simple structure of the filter, the Kalman filter is widely used in multi-source data fusion [8]. The current radar surveillance information processing system uses adaptive Kalman filter technology (AKF), which can prevent filter divergence and improve the realtime performance of the system [9]. However, Kalman filtering also has shortcomings in filtering accuracy and stability in the field of aviation surveillance, such as: poor dynamic filtering performance [10], high computational complexity. In the maneuvering state, the Kalman filter has low fusion precision and the model tuning process is cumbersome. This paper proposes an aerial surveillance information fusion system based on recurrent neural network to solve the problems encountered by the Kalman filter algorithm.

Because the neural network has a strong ability to fit nonlinear relationships [11], and does not have to dynamically update the model during use like Kalman filtering. In various neural networks, the recurrent neural network is suitable for processing sequence data [12], which is more consistent with the continuity of flight track data. Therefore, we choose recurrent neural network as the aviation surveillance information fusion model. And for the problem that the Kalman filter has a slow update of the state equation in the maneuvering state of the aircraft, this paper uses the recurrent neural network to judge the maneuvering state, thus assisting the Kalman filtering and other fusion methods to detect the maneuvering state in time. The dataset used in this paper is the monitoring data of 29 secondary radars in a certain region of China and the ADS-B data of corresponding time periods in the region. Since the real flight calibration data cannot be obtained, and the ADS-B data has higher precision than the radar data, ADS-B is selected as the fusion target data, and the data of multiple radars is the original aviation monitoring information. This paper briefly introduces the specific implementation of the discriminant algorithm and the fusion algorithm. The experimental results of the algorithm are given and analyzed.

\section{Algorithm}

\subsection{Recurrent neural network}

Traditional neural networks can only process a single input, and the previous input and the latter input are completely uncorrelated. However, recurrent neural network is a kind of neural network for processing sequence data. Sequence data usually refers to the data collected at consecutive time points. This kind of data reflects the changing state of a certain thing over time, and its characteristics are related to the front and back. The aviation monitoring information of each monitoring source for a specific flight target is such time series data.

The key to recursive neural networks to solve timing data problems is that their hidden layer state preserves previous input information. The structure of the recurrent neural network is different from the traditional multi-layer perceptron, and its input layer interacts with the signal from the hidden layer of the previous element in the sequence to the current hidden layer. In the recurrent neural network, since the input is superimposed with the historical signal, the reverse conduction is different from the traditional neural network, because for the input layer at a specific moment, the residual is not only from the output, but also from the hidden layer. BPTT (back-propagation through time) algorithm is a commonly used method for training recurrent neural networks. Its essence is BP algorithm, except that recurrent neural network processes time series data. Its back propagation is time-based, so it is called back-propagation through time. . The central idea of BPTT is the same as the BP algorithm, that is, the gradient descent method, which continuously searches for a better point along the negative gradient direction of the parameter to be optimized until convergence.

Recurrent neural networks are often used in text classification, machine translation, speech recognition and other fields. This topic uses recurrent neural networks to process monitoring source information, which is an innovation in application scenarios. 


\subsection{Long and short term memory network}

The core capability of a recurrent neural network is that it can connect previous information to the current task. When the interval between the relevant information and the current location is small, the network can learn the previous information. However, as the interval between relevant information and predicted location increases, the network will gradually lose the ability to learn such far away information. In order to solve this long-term dependence problem, Long and short term memory network came into being, referred to as LSTM, which is a special recurrent neural network model. The core idea of LSTM is the cell state, and the horizontal line runs through the top of the figure. By designing the structure of the "gate", LSTM interacts with the state of the cell to remove or add information to the state of the cell. A "gate" is a method of selectively passing information, including a sigmoid neural network layer and a bitwise multiplication operation. The LSTM has a total of three gates that in turn determine what information is discarded from the cell state, what new information is stored in the cell state, and what cell state is output from the LSTM unit. The presence of some interfering signals in the track data processed by this subject will adversely affect the effectiveness of the discriminant algorithm and the fusion algorithm, which can be reduced by the use of LSTM.

\subsection{Weighted least squares method}

The least squares method finds the best function match of the data by minimizing the square of the error. Curve fitting can be performed using the least squares method. In the process of fitting the curve, the general least squares method treats the importance of each data in the time series equally, but in fact, the influence of the time series data on the future should be different. In general, recent data has a greater impact on the future than long-term data. Therefore, a more reasonable method is to use the weighting method to assign a larger weight to the recent data and a smaller weight to the forward data.

\section{Proposed Method}

\subsection{Overall framework}

The aviation monitoring information fusion subsystem consists of four parts, namely, a preprocessing module, a maneuvering state discriminating module, a flight position prediction module, and a fusion module. The preprocessing includes message parsing, coordinate conversion, and track matching. The message parsing is to convert the original message of the radar and ADS-B into data including various monitoring information, and the coordinate conversion is to convert the position information of the aircraft into Geodetic Rectangular coordinates, track matching is used to combine scattered points into tracks. The maneuvering state discriminating module discriminates whether the aircraft is in a maneuver state by using a recurrent neural network, and the discriminating result is used for one-dimensional input of the fusing process. The flight position prediction module uses the continuous point trace to fit the flight curve based on the weighted least squares method, and predicts the future flight position, and the position information of each radar observation can be unified to the same time for fusion. The fusion module uses the recurrent neural network to fuse the monitoring information of the multi-radar to the flight position of the aircraft at the current time.

\subsection{Maneuvering state discrimination algorithm based on recurrent neural network}

Since the fusion effect of Kalman filter in the maneuvering state of the aircraft is not ideal, the method constructs a recurrent neural network model to identify the maneuvering state. According to the data of single radar or ADS-B, it is calculated whether the target aircraft is in a maneuver state (maneuver mainly refers to the change of flight state with time, such as turning, altitude climbing, speed change), so that the fusion algorithm can be adjusted in time when the aircraft is in a maneuver state. 


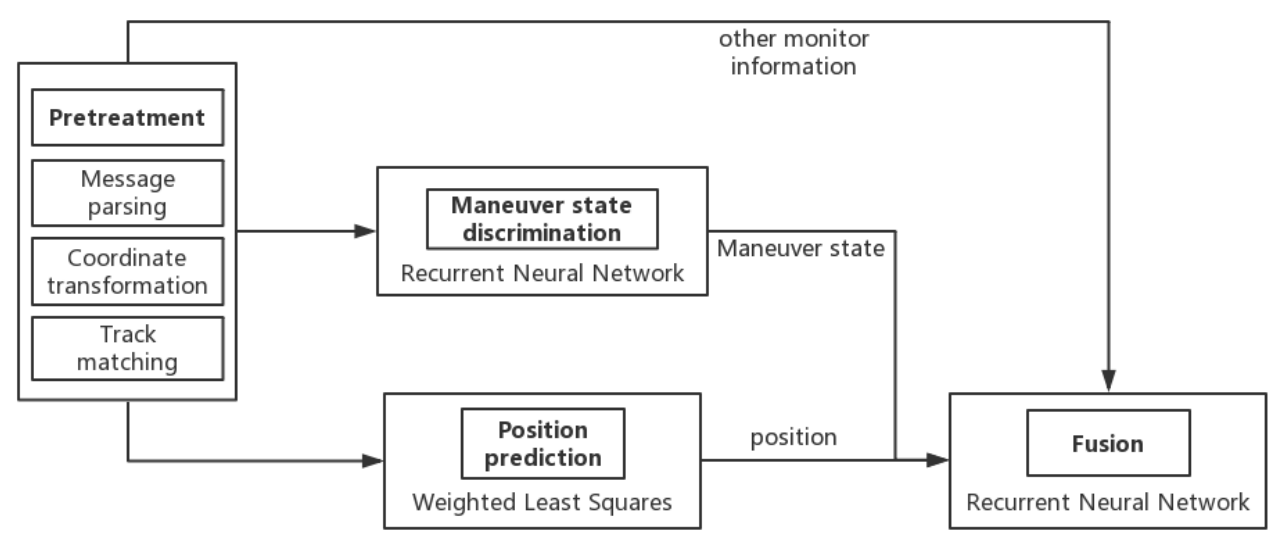

Fig. 1: Overall framework.

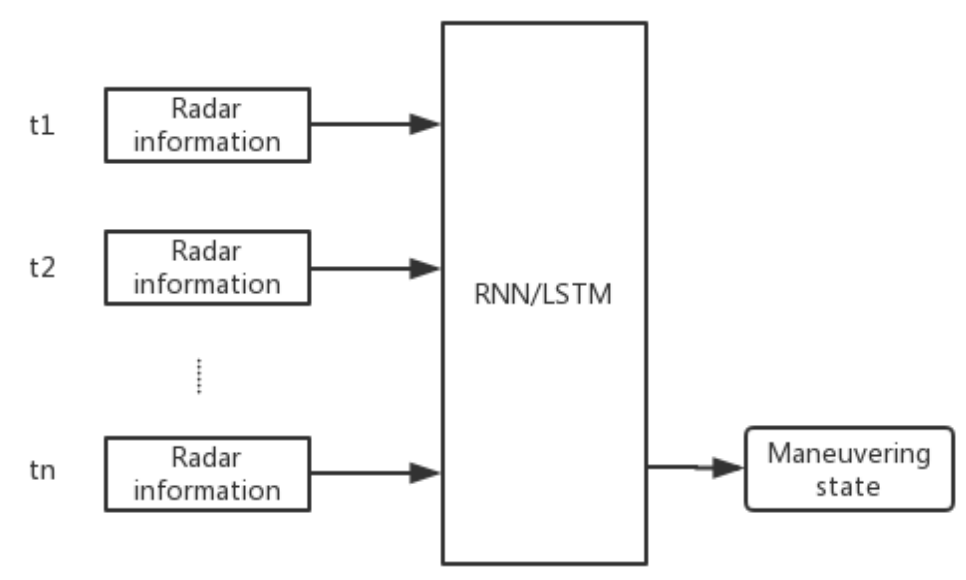

Fig. 2: Maneuver state discrimination model.

As shown in Fig 2, the method uses the monitoring information of the aircraft at the first $\mathrm{n}$ times to predict the maneuvering state of the aircraft at the nth time, which is a two-class problem. In order to improve the accuracy of the determination of the maneuvering state, the method transforms the monitoring information on the basis of the original data. The selected features include the time interval, the displacement in each direction of the geodetic coordinate system, the height change value, the speed and the distance between the aircraft and the source of the surveillance. A more accurate description of the position of the aircraft is achieved. And experiment with different models (RNN, LSTM) and different training steps.

In order to obtain the label of the classification problem, it is necessary to manually mark whether the aircraft in the selected data is in a maneuver state. The turning part is intercepted by visualizing the flight path; the speed and height changes are set to a certain threshold for screening.

\subsection{Position prediction based on weighted least squares}

At present, the Kalman filter used by the monitoring system for information fusion does not need time alignment, because the Kalman filter algorithm corrects the monitoring information while predicting, thereby completing the process of information fusion. But on the contrary, this paper divides the process of prediction and fusion into two steps. Since the current research scenario requires model training for each monitoring source data at the same time, time alignment must be performed in the data preprocessing step. The time alignment needs to predict the information of a specific moment according to the current aircraft history monitoring information for each monitoring source. Due to changes in maneuverability, linear functions cannot be used to fit the relationship between monitoring information and time.

In this paper, the least square method is used to predict the monitoring information. Firstly, the motion model of the aircraft should be considered. For the motion state of the aircraft in one direction, the following three motion equations can be used to describe (X direction): 
1) Uniform linear motion: $a t+b=X$

2) Uniform shift linear motion: $a t^{2}+b t+c=X$

3) Uniform acceleration linear motion: $a t^{3}+b t^{2}+c t+d=X$

These three motion states are easy to understand. Civil aviation aircraft can maintain a uniform circular motion during cornering. Therefore, under this premise, the speed change of the aircraft in the $\mathrm{x}$ direction is related to the angle of rotation. The motion of the turning can be approximated by a one-dimensional cubic equation. The accuracy is sufficient. By using the least squares method, the curve with the smallest sum of squared errors is selected, and the monitoring information at a specific time is obtained according to the curve equation.

Since recent data has a greater impact on predictions than forward data, this paper uses weighted least squares. For the weight function of the weighted least squares method, choose the following form:

$$
w\left(x_{i}\right)=\theta^{i}(1+s)
$$

In Equation 1, $\theta$ is a number in the range of $(0,1)$, and the newer data has a smaller $i$ value. The method is fitted with 10 historical data, $x_{0}$ represents the latest radar data, the weight is the largest, $x_{9}$ represents the oldest data, and the weight is the smallest. Since the maneuver state has a greater impact on the future, $\mathrm{s}$ in Equation 2-3 is 1 when the aircraft is in a maneuver state, whereas s is 0 .

In this paper, different values of $\theta$ are selected for experiments. When $\theta=5 \times 10^{-9}$, the prediction error is the smallest, about 104 meters.

\subsection{Multi-radar monitoring information fusion algorithm based on recurrent neural network}

In view of the shortcomings of Kalman filter algorithm used for aerial surveillance information fusion (requiring repeated calibration flights and expert parameter adjustment), this paper adopts the method based on recurrent neural network to fuse multi-radar surveillance information. At present, there is no flight calibration data. Because ADS-B data is the information sent by airborne equipment to ADS-B ground station, it has a high accuracy without tampering, so ADS-B data is temporarily taken as the accurate monitoring information of aircraft. The RNN model is trained with the corresponding ADS-B surveillance information as the target by using the current surveillance information of multiple radars for the same aircraft. Through this algorithm, the position of the aircraft can be predicted efficiently in the geodetic rectangular coordinate system. The algorithm predicts the position of two directions in the geodetic rectangular coordinate system.

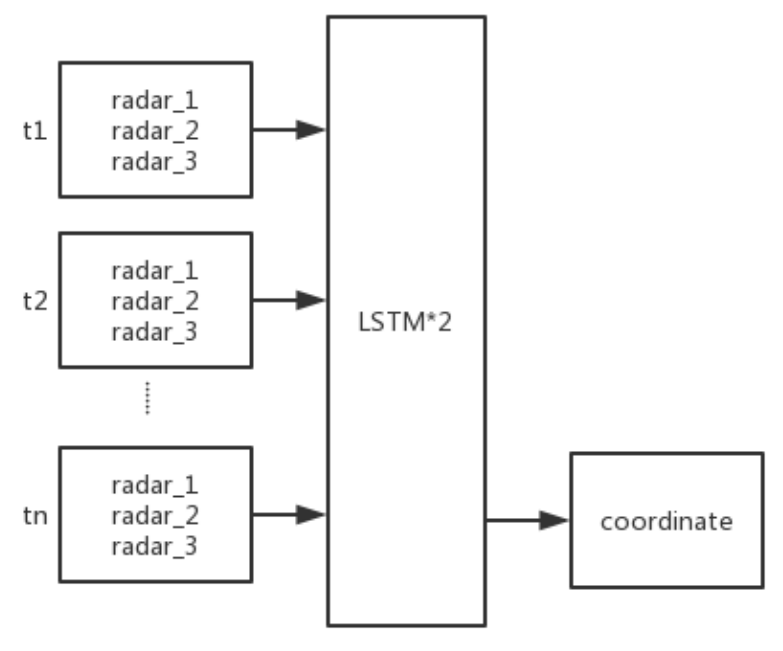

Fig. 3: Radar Information Fusion Model.

As shown in Figure 3, this experiment uses the monitoring information of radars 1 to 3 to train the recurrent neural network, and uses the model to predict the position of the aircraft in various directions under the geodetic coordinate system. A total of 50 tracks were selected for a total of 100,000 points. Experiments were performed on different features, different RNN models, and different training parameters. 
The feature selection process is mainly to convert the original data into a maneuver state, a time gap, a distance from the radar, and a geodetic coordinate as the input of the neural network. The basic RNN and the LSTM with gate structure are selected. Finally, the monitoring information for the flight altitude above 6000 meters is tested separately. The reason for selecting 6000 meters is that there are more maneuvers in the 6000 meters or less. In the area above 6000 meters, the aircraft enters the leveling layer, the maneuvering state is reduced, and the prediction difficulty is reduced ).

\section{Illustration Results}

\subsection{Judgment results based on RNN}

In order to verify that the recursive neural network-based maneuver state judgment algorithm and the recurrent neural network-based aeronautical surveillance information fusion algorithm are more accurate, this paper selects two kinds of recurrent neural networks: Recurrent Neural Network (referred to as RNN). And Long Short Term Memory (LSTM) as an experimental model.

The RNN-based maneuvering state discrimination algorithm uses 12,000 points of the No. 1 radar for training and testing. The accuracy of the judgment of the maneuvering state in the experimental results is shown in Table 1:

Table 1: Maneuvering state judgment accuracy

\begin{tabular}{|c|c|c|c|c|c|c|}
\hline Model & \multicolumn{3}{|c|}{ RNN } & \multicolumn{3}{c|}{ LSTM } \\
\hline Training Step & 5 & 10 & 20 & 5 & 10 & 20 \\
\hline $\mathrm{x}, \mathrm{y}, \mathrm{h}, \mathrm{t}$ & 0.217 & 0.273 & 0.290 & 0.291 & 0.297 & 0.263 \\
\hline$\Delta x, \Delta y, \Delta h, \Delta t$ & 0.751 & 0.773 & 0.751 & 0.743 & 0.786 & 0.693 \\
\hline$\Delta x, \Delta y, \Delta h, \Delta t, \mathrm{v}, \mathrm{dis}$ & 0.853 & 0.920 & 0.897 & 0.839 & 0.959 & 0.910 \\
\hline
\end{tabular}

In Table 1, $\mathrm{x}$ and $\mathrm{y}$ represent the horizontal and vertical coordinates of the aircraft in the Cartesian coordinate system, $\Delta \mathrm{x}$ and $\Delta \mathrm{y}$ represent the displacements in their respective directions, $\mathrm{h}$ is the flight height of the aircraft, and $\Delta \mathrm{h}$ is the height variation. $\mathrm{t}$ is time, $\Delta \mathrm{t}$ is the time interval from the previous moment, $\mathrm{v}$ is the speed, and dis is the distance between the aircraft and the monitoring source. Through comparison experiments, it can be known that the discriminating accuracy is higher when the displacement amount, height climbing amount, speed, and time interval are selected as inputs. According to Table 4-1, the accuracy of the judgment of the maneuvering state after using the LSTM is generally higher than that of using the RNN, and the effect is best when the step size is taken 10 times.

\subsection{Fusion results based on RNN}

The aeronautical surveillance information fusion algorithm based on recurrent neural network can obtain more accurate position information than the radar data when the optimized features are selected. The neural network input features selected by this method include position, altitude, time interval, maneuver status and target. The distance from the radar. The neural network target output is the target location information in the ADS-B data. Multiple sets of comparative experiments were performed using a double layer LSTM with a step size of 10 .

First, select part of the radar data of No. 1 to No. 3 and the corresponding ADSB data, a total of 50 tracks, 100000 points, and select 10 tracks as test sets. According to statistics, the average error of radar data in the whole data set is about 271 meters. The following table is an error statistic for experiments with different characteristics and data sets at different flight altitudes(p represents the position in the Cartesian coordinate system; a represents altitude; ms represents maneuver status; $t$ represents the number of milliseconds from the day 00:00:00; ti represents the number of milliseconds from the previous moment; $\mathrm{d}$ represents the distance from the radar):

Table 2: Monitoring information fusion result error

\begin{tabular}{|c|c|c|c|}
\hline & p,a,t & p,a,ms,ti & p,a,ms,ti,d \\
\hline All & $1129 \mathrm{~m}$ & $443 \mathrm{~m}$ & $202 \mathrm{~m}$ \\
\hline Below 6000 & $950 \mathrm{~m}$ & $192 \mathrm{~m}$ & $121 \mathrm{~m}$ \\
\hline Above 6000 & $1573 \mathrm{~m}$ & $515 \mathrm{~m}$ & $326 \mathrm{~m}$ \\
\hline
\end{tabular}


It can be seen from Table 2 that it is best to select the time interval as the feature and add the radar distance and maneuver status as the supplementary feature, and the error of the fusion result in the region below 6000 meters is smaller than the height above 6000 meters. However, due to the low error of radar data in low-altitude conditions, in fact, compared with radar, the result of fusion at 6000 meters is closer to the real result; in the region below 6000 meters, the result of fusion is sometimes worse than a single Original monitoring information. However, none of the radars can guarantee that the error is smaller than the fusion result in the entire track, and the fusion result error is smaller than the average of the data errors of the monitoring information sources.

The following two figures are a comparison of position information after fusion, position information of radar observations, and real position information.

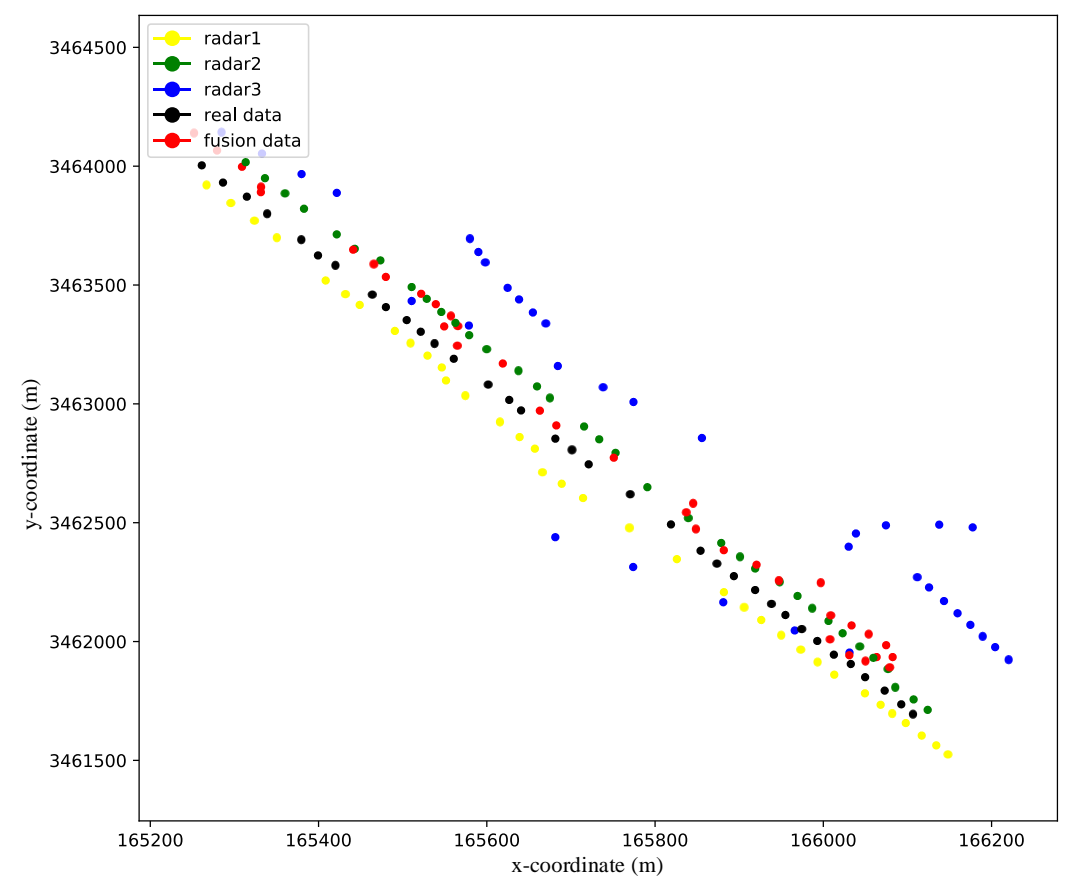

Fig. 4: Fusion result and origin data.

Fig. 4 shows the observation position of the radar No. 1-3, the real target position, and the position after the fusion. It can be seen from the observation that there is a case where the position of the radar observation is far from the real position (such as radar3), but the fusion can avoid such a large error.

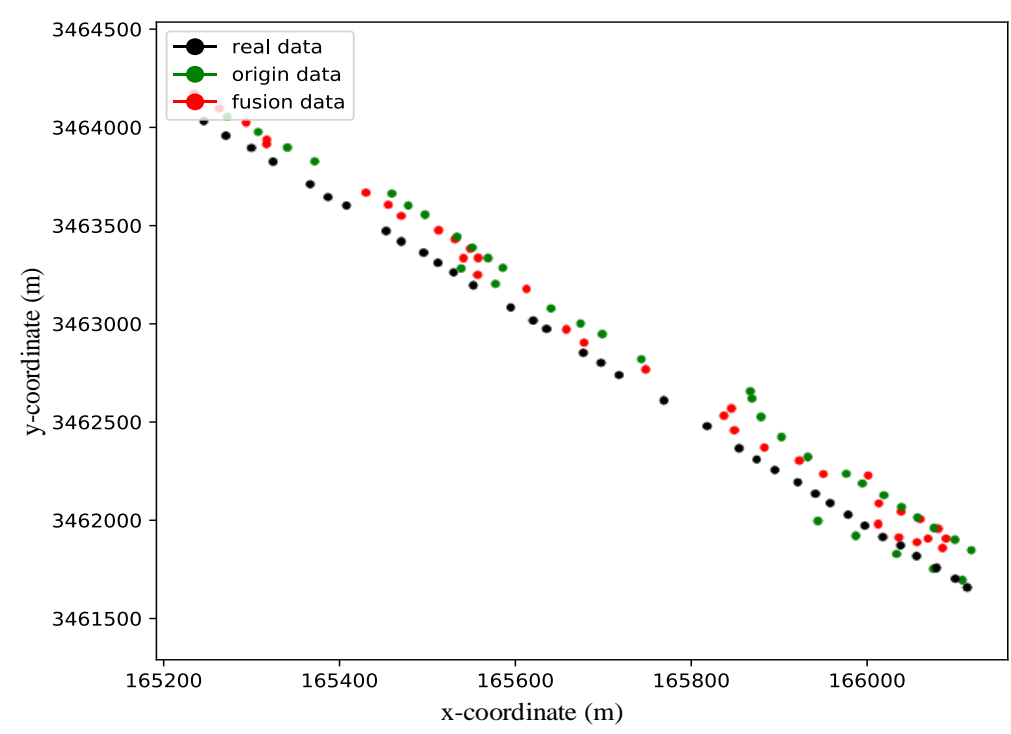

Fig. 5: Fusion result and means of origin data. 
In Fig. 5, the mean value of the radar observation information, the real target position, and the position after the fusion. It can be seen that in most cases, the merged position is closer to the real position than the radar observation position average.

\section{Conclusions and Discussion}

As shown by the experimental results, the recursive neural network-based motor state discriminant model has a $95.9 \%$ discriminant accuracy, which can accurately identify the maneuver state, and the maneuver state as the input feature of the fusion model has a positive impact on the fusion result. The error of the fusion result obtained by the aeronautical surveillance information fusion technology based on recurrent neural network can reach about 202 meters, and the average error of the radar is about 271 meters, so the error can be reduced by nearly 70 meters. In the low air below 6000 meters, the error after fusion is about 121 meters. In the future work, we need to avoid the influence of bad data on the fusion result as much as possible, so that the monitoring information fusion algorithm based on recurrent neural network can continue to reduce the error.

\section{Acknowledgments}

The authors acknowledge the National Key R\&D Program of China (Grant: 2016YFB0502400), the National Key R\&D Program of China (Grant: 2016YFB0502404).

\section{References}

[1] ZHOU Jun, "Kalman Filtering of Radar Technology in Air Traffic Control Surveillance System," Science \& Technology Vision, Jul. 2015, pp. 65-65, 155, doi:10.3969/j.issn.2095-2457.2015.19.048.

[2] Olivier B , Pierre G , Nicolas, et al. Multi Sensor Data Fusion Architectures for Air Traffic Control Applications[M]// Sensor and Data Fusion. InTech, 2009.

[3] Hall D L, Llinas J. An introduction to multisensor data fusion[J]. Proceedings of the IEEE, 2002, 85(1):6-23.

[4] Donohue G . Vision on aviation surveillance systems[C]// IEEE International Radar Conference. IEEE, 1995.

[5] Cheng Jian. Improvement method of measurement accuracy of air traffic secondary radar [J]. Electronic Technology and Software Engineering, 2018.

[6] Li Zijun. The Principle and Future Development and Application of ADS-B Broadcast Automatic Correlation Monitoring[J]. Journal of Civil Aviation Flight University of China, 2008, 19(5): 11-14.

[7] Brookner, Eli. Tracking and Kalman Filtering Made Easy[J]. 1998, 10.1002/0471224197.

[8] Hu Hongcan, Guo Li, Zhu Junzhu. Application of Joint Kalman Filter in Data Fusion[J]. Fire Control Radar Technology, 2005, 34(1): 1-4.

[9] YANG Yong-jian, FAN Xiao-guang, and WANG Sheng-da, "Target tracking based on amendatory Kalman filter," Systems Engineering and Electronics, May 2014, pp. 846-851, doi:10.3969/j.issn.1001-506X.2014.05.06.

[10] Liu Guohai, Li Yuxue, Shi Wei, et al. Application of dynamic Kalman filter in state estimation of navigation test[J]. Chinese Journal of Scientific Instrument, 2009, 30(2).

[11] Zhai D , Li L , Jin F . Fuzzy neural network for nonlinear-systems model identification[J]. Chinese Journal of Computers, 2004, 3(4):1282-1287 vol.3.

[12] Nallapati R , Zhai F , Zhou B . SummaRuNNer: A Recurrent Neural Network based Sequence Model for Extractive Summarization of Documents[J]. 2016. 